\title{
Erratum to: New high precision measurements of the cosmic charged particle rate beyond the Arctic Circle with the PolarquEEEst experiment
}

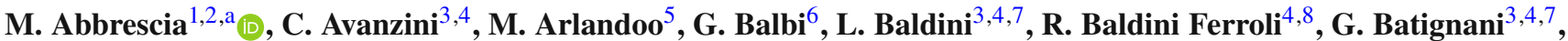 \\ M. Battaglieri ${ }^{4,9,10}$, S. Boi ${ }^{4,11,12}$, D. Cavazza ${ }^{6}$, E. Bossini ${ }^{13}$, F. Carnesecchi ${ }^{4,6,14}$, C. Cicalò ${ }^{4,12}$, L. Cifarelli ${ }^{4,6,14}$, \\ F. Coccetti ${ }^{4}$, E. Coccia ${ }^{4,15}$, A. Corvaglia ${ }^{4,16}$, D. De Gruttola ${ }^{17,18}$, S. De Pasquale ${ }^{17,18}$, F. Fabbri ${ }^{4,8}$, D. Falchieri ${ }^{6}$,

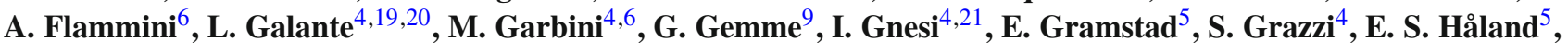 \\ D. Hatzifotiadou ${ }^{4,6,13}$, P. La Rocca ${ }^{4,22,23}$, Z. Liu ${ }^{24}$, L. Lombardo ${ }^{25}$, G. Mandaglio ${ }^{4,23,26}$, A. Margotti ${ }^{6}$, G. Maron ${ }^{27}$, \\ M. N. Mazziotta ${ }^{2, b}$, S. Meneghini' ${ }^{6}$, S. A. Millerjord ${ }^{5}$, A. Mulliri ${ }^{11,12}$, R. Nania $^{4,6}$, F. Noferini ${ }^{4,6, c}$, F. Nozzoli ${ }^{28}$, \\ F. Ould-Saada ${ }^{5}$, F. Palmonari ${ }^{4,6,14}$, M. Panareo ${ }^{16,29}$, M. P. Panetta ${ }^{4,16}$, R. Paoletti ${ }^{3,30}$, M. Parvis ${ }^{25}$, C. Pellegrino ${ }^{4,27}$,

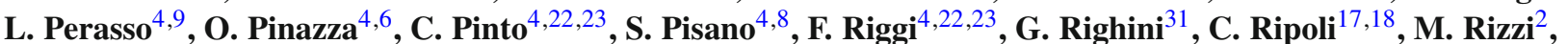 \\ G. Sartorelli ${ }^{4,6,14}$, E. Scapparone ${ }^{4,6}$, M. Schioppa ${ }^{21,32}$, G. Scioli ${ }^{6,14}$, A. Scribano ${ }^{30}$, M. Selvi ${ }^{4,6}$, G. Serri ${ }^{4,11,12}$,

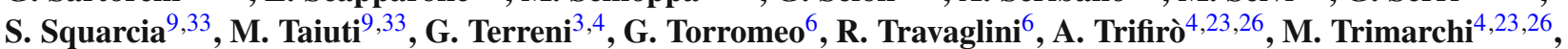 \\ C. Veri ${ }^{6}$, C. Vistoli $^{27}$, L. Votano ${ }^{34}$, M. C. S. Williams ${ }^{4}$, A. Zichichi ${ }^{4,6,13,14}$, R. Zuyeuski ${ }^{4}$ \\ ${ }^{1}$ Dipartimento Interateneo di Fisica, Università di Bari, Bari, Italy \\ 2 INFN Sezione di Bari, Bari, Italy \\ ${ }^{3}$ INFN Sezione di Pisa, Pisa, Italy \\ ${ }^{4}$ Museo storico della Fisica e Centro studi e ricerche "E. Fermi”, Roma, Italy \\ ${ }^{5}$ Physics Department, Oslo University, Oslo, Norway \\ ${ }^{6}$ INFN Sezione di Bologna, Bologna, Italy \\ ${ }^{7}$ Dipartimento di Fisica, Università di Pisa, Pisa, Italy \\ ${ }^{8}$ INFN, Laboratori Nazionali di Frascati, Frascati (RM), Italy \\ ${ }^{9}$ INFN Sezione di Genova, Genova, Italy \\ 10 Thomas Jefferson National Accelerator Facility, Newport News, VA 23606, USA \\ ${ }^{11}$ Dipartimento di Fisica, Università di Cagliari, Cagliari, Italy \\ 12 INFN Sezione di Cagliari, Cagliari, Italy \\ ${ }^{13}$ CERN, Geneva, Switzerland \\ ${ }_{15}^{14}$ Dipartimento di Fisica, Università di Bologna, Bologna, Italy \\ ${ }^{15}$ Gran Sasso Science Institute, L'Aquila, Italy \\ ${ }^{16}$ INFN Sezione di Lecce, Lecce, Italy \\ ${ }^{17}$ Dipartimento di Fisica, Università di Salerno, Salerno, Italy \\ ${ }^{18}$ INFN Gruppo Collegato di Salerno, Salerno, Italy \\ ${ }^{19}$ Dipartimento di Scienze Applicate e Tecnologia, Politecnico di Torino, Torino, Italy \\ ${ }^{20}$ INFN Sezione di Torino, Torino, Italy \\ ${ }^{21}$ INFN Gruppo Collegato di Cosenza, Laboratori Nazionali di Frascati (RM), Frascati, Italy \\ ${ }^{22}$ Dipartimento di Fisica e Astronomia, Università di Catania, Catania, Italy \\ ${ }^{23}$ INFN Sezione di Catania, Catania, Italy \\ 24 ICSC World laboratory, Geneva, Switzerland \\ ${ }^{25}$ Dipartimento di Elettronica e Telecomunicazioni, Politecnico di Torino, Torino, Italy \\ ${ }^{26}$ Dipartimento di Scienze Matematiche e Informatiche, Scienze Fisiche e Scienze della Terra, Università di Messina, Messina, Italy \\ ${ }^{27}$ INFN-CNAF, Bologna, Italy \\ ${ }^{28}$ INFN Trento Institute for Fundamental Physics and Applications, Trento, Italy \\ ${ }^{29}$ Dipartimento di Matematica e Fisica, Università del Salento, Lecce, Italy \\ ${ }^{30}$ Dipartimento di Scienze Fisiche, della Terra e dell'Ambiente, Università di Siena, Siena, Italy \\ ${ }^{31}$ CNR, Istituto di Fisica Applicata "Nello Carrara", Sesto Fiorentino, Italy \\ 32 Dipartimento di Fisica, Università della Calabria, Rende, CS, Italy \\ ${ }_{33}$ Dipartimento di Fisica, Università di Genova, Genova, Italy \\ ${ }^{34}$ INFN, Laboratori Nazionali del Gran Sasso, Assergi, AQ, Italy
}


Erratum to: Eur. Phys. J. C (2020) 80:665

https://doi.org/10.1140/epjc/s10052-020-8213-2

In the original version of this article, the names of four authors were missing in the author list: L. Perasso, O. Pinazza, C. Pinto and S. Pisano.

The original article has been corrected.

\section{Acknowledgements Funded by SCOAP3.}

Open Access This article is licensed under a Creative Commons Attribution 4.0 International License, which permits use, sharing, adaptation, distribution and reproduction in any medium or format, as long as you give appropriate credit to the original author(s) and the source, provide a link to the Creative Commons licence, and indicate if changes were made. The images or other third party material in this article are included in the article's Creative Commons licence, unless indicated otherwise in a credit line to the material. If material is not included in the article's Creative Commons licence and your intended use is not permitted by statutory regulation or exceeds the permitted use, you will need to obtain permission directly from the copyright holder. To view a copy of this licence, visit http://creativecommons.org/licenses/by/4.0/.

The original article can be found online at https://doi.org/10.1140/ epjc/s10052-020-8213-2.

a e-mail: marcello.abbrescia@ba.infn.it (corresponding author)

b e-mail: Marionicola.Mazziotta@ba.infn.it (corresponding author)

c e-mail: Francesco.Noferini@bo.infn.it (corresponding author) 